\title{
Epigenetic abnormalities and therapies for hematological malignancies
}

\author{
Toshio Kitamura ${ }^{1}$
}

Received: 17 June 2019 / Accepted: 17 June 2019 / Published online: 6 July 2019

(c) Japanese Society of Hematology 2019

The 9th JSH International Symposium was held in Kyoto on July 26-27, 2018. The meeting focused on "Epigenetic abnormalities and therapies of hematological malignancies," and included numerous engaging and informative talks by prominent speakers from Asia, Europe, and the United States. As in the past, we organized ASH-JSH and EHA-JSH Joint Symposia, as well as two simultaneous sessions with Asian scholars. In addition to the 19 talks by invited speakers from abroad and 11 by Japanese presenters, 67 poster presentations were accepted, including three that were selected for oral presentation.

In addition to the academic presentations, the rock band Negative Selection, consisted of immunology and hematology professors held a gig at the evening reception, joined by friends from around the world, including Margaret (Peggy) Goodell (USA) and Radek Skoda (EU) on guitar, and Issay Kitabayashi and Ai Kotani (Japan) on piano. Dr. Kotani and Xiaoxiao Liu, a graduate student from China in my lab, also sang as background chorus. After the meeting, we had a fantastic excursion to the temples Ryoan-ji and Kinkaku-ji (The Temple of the Golden Pavilion), a few hours before a big typhoon reached Kyoto. Our special visit into the Golden Pavilion was particularly unforgettable. To commemorate this symposium, I invited four of the invited speakers to write reviews on "Epigenetics of hematological malignancies" in "Progress in Hematology".

Dr. Goodell gave an excellent talk on NPM1 mutations and efficient gene editing techniques using blood cells. In this "Progress in Hematology", Dr. Goodell and colleagues present a comprehensive and insightful review on the mutations of nucleophosmin 1 (NPM1) (Brunetti et al.). Mutation of NPM1 is one of the most frequent mutations identified

Toshio Kitamura

kitamura@ims.u-tokyo.ac.jp

1 Institute of Medical Science, University of Tokyo, Tokyo, Japan in AML, most of which are frameshift mutations of the C-terminal domain with a 4-bp insertion. These mutations disrupt the nucleolar localization signal of NPM1, and create a new nuclear export signal (NES), resulting in its cytoplasmic localization. Mutant NPM1 is thus usually called NPM1c. NPM1 is reported to play a broad spectrum of roles in relation to many distinct biological functions of cells, but despite extensive research into NPM1c, it remain unclear what role it plays in leukemogenesis. The review of Brunetti et al. summarizes the current state of knowledge about NPM1 and its mutant forms, and should inspire new thinking on its functions in the context of hematologic malignancies.

Dr. Atsushi Iwama and colleagues describe roles of polycomb complex PRC1 and PRC2 in myelodysplastic syndromes (MDS) and myeloproliferative neoplasm (MPN). While EZH2, a catalytic component of PRC2, acts as an oncogene in solid cancers and some lymphomas, in MDS it acts as a tumor-suppressor. In this review, the authors focus on mutations of EZH2, EED, and Suz12 of PRC2 and BCOR of PRC1, as well as ASXL1 which cooperates with PRC1 in de-ubiquitination of histone H2AK119ub, and and with PRC2 in trimethylation of histone H3K27. The authors also discuss molecular targeted therapies of MDS and MPN using EZH1/2 inhibitors, BRD4 inhibitors, and DNA hypomethylating agents (HMA).

Dr. Shuhei Asada from my group describes the roles of ASXL1 in modification of a representative active mark, H3K4me3, and a representative suppressive mark, $\mathrm{H} 3 \mathrm{~K} 27 \mathrm{me}$, as well as de-ubiquitination of H2AK119Ub. Mutations of ASXL1 are observed in various myeloid malignancies, and always associated with poor prognosis. ASXL1 mutations occur at the $5^{\prime}$ end of the last exon, leading to its C-terminal truncation, suggesting that ASXL1 mutations are gain-of-function or dominant-negative mutations. In fact, ASXL1 mutations repress expression of ID3 and TJP1, which are related to erythroid differentiation via decreased H3K4me, de-repress expression of HoxA9/10 and miR125a, 


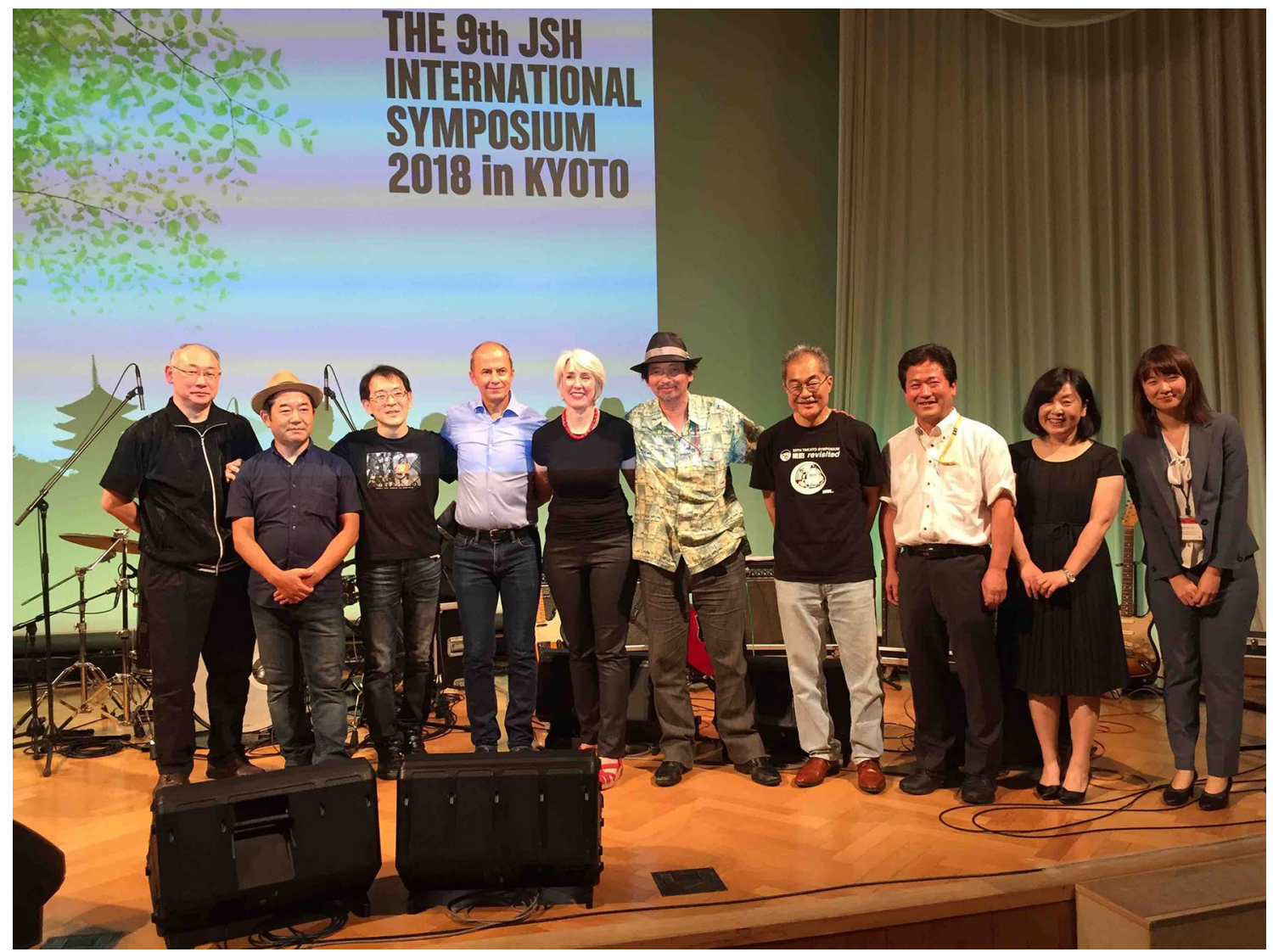

Band members and guest performers after the small concert at the Symposium party

which are involved in leukemogenesis via decreased H3K27me3, and increase expression of HoxA5/7/9 as well as IRF8 via de-ubiquitination of H2AK119Ub. Thus, disruption of normal function of ASXL1 results in disordered control of histone modification, leading to myeloid malignancies. Dr. Asada summarizes the current state of knowledge about functions of ASXL1 mutations reported by several research groups.

Hypomethylating agents, including azacitidine and decitabine, show some efficacy in patients with MDS and CMML. These agents are also used for elderly patients with AML, although with limited efficacy. However, the molecular mechanisms underlying the effects of HMA on myeloid malignancies remain elusive. Drs. Duchmann and Itzykson summarize many aspects of HMA therapies on myeloid malignancies, including approved therapeutic uses, clinical trials, the search for biomarkers, new regimens, new oral formulations of HMA, and combination therapy with HDAC inhibitors, lenalidomide, an oral AraC, a Bcl2 inhibitor, IDH inhibitors, and an inhibitor of NEDD8-activating enzyme. Thus, this review provides an excellent overview of the current status and prospects of HMA therapy.

Happily, the major typhoon that was approaching Kyoto and Tokyo slowed down enough to let us complete the JSH 


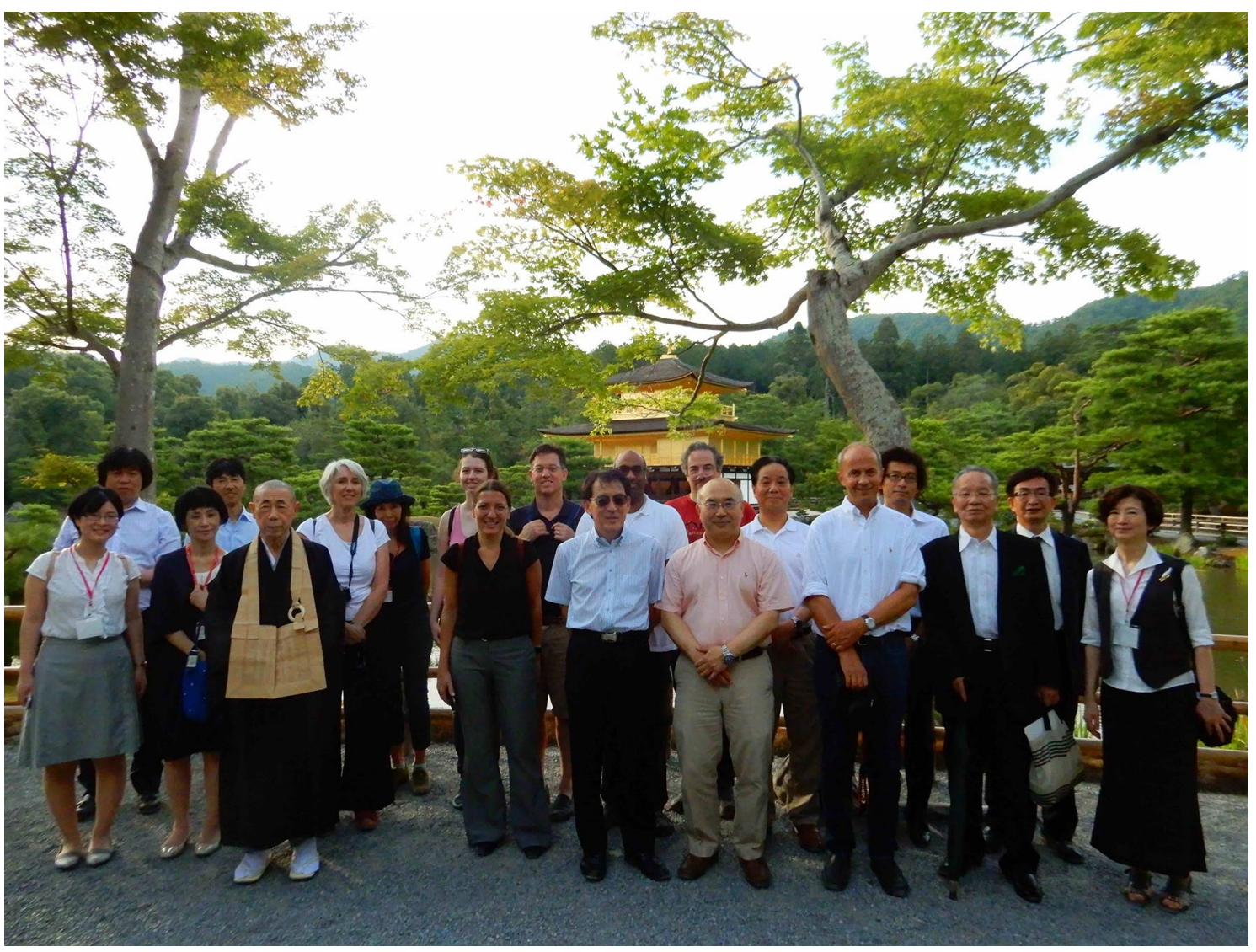

Excursion to Kinkaku-ji Temple just before the typhoon arrived

International meeting, while still providing a refreshing breath of cool during a hot Kyoto summer and the warmth of the many discussions during the meeting. Late on the night of the final day, bullet trains were stopped between Kyoto and Tokyo due to the typhoon. Fortunately, none of the meeting participants appear to have suffered from the weather.

I hope that the four reviews posted in this Progress in Hematology feature will help IJH readers gain a better understanding of the current state of basic research, ongoing clinical trials, and approved therapies for hematological malignancies caused by epigenetic abnormalities.

Publisher's Note Springer Nature remains neutral with regard to jurisdictional claims in published maps and institutional affiliations. 